\title{
Proceeding
}

Supplementary Issue: Spring Conferences of Sports Science. 15th Convention and Workshop of the International Network of Sport and Health Science, 5-8 June 2019. University of Las Palmas de Gran Canaria, Las Palmas de Gran Canaria, Spain.

\section{A specific training protocol for the elderly}

\author{
SALVATORE PIGNATO , ANTONELLA QUINTO, SEBASTIANO GAGLIARDI, VITTORIA PATANIA \\ University Kore of Enna, Italy
}

\begin{abstract}
It is widely known that in elderly people a regular physical activity of mild intensity shows significant benefits in term of health, while a sedentary lifestyle contributes, together with other factors of risks, to develop some chronic degenerative diseases, particularly cardiovascular metabolic and osteo-articular diseases. Therefore, the aim of this study is useful to assess influence of controlled training, based on conditional and coordinative motor abilities, in elderly people. The group of this study is made up of twenty members (experimental and control groups) aged from 65 to 75 . The experimental groups have trained for nine months ( 2 times for week with 45-60' for each training session) and no training for control group. Before and after nine months of training, each participant has been assessed with the tests presented in the article. There were no significant differences for the control group in the pre and post comparison except for the Chair-Stand test characterized by a worsening of the results. On the contrary, there were statistically significant differences between the pre and post-test evaluations of the experimental group, in which improvements have been shown in the test. This investigation showed the training benefit on long period in elderly without any compromising. Keywords: Elderly people; Tinetti Test; Falls; Motility.
\end{abstract}

\section{Cite this article as:}

Pignato, S., Quinto, A., Gagliardi, S., \& Patania, V. (2019). A specific training protocol for the elderly. Journal of Human Sport and Exercise, 14(4proc), S638-S645. doi:https://doi.org/10.14198//hse.2019.14.Proc4.23

Corresponding author. University Kore of Enna, Italy.

E-mail: salvatore.pignato@unikore.it

Supplementary Issue: Spring Conferences of Sports Science. 15th Convention and Workshop of the International Network of Sport and Health Science, 5-8 June 2019. University of Las Palmas de Gran Canaria, Las Palmas de Gran Canaria, Spain. JOURNAL OF HUMAN SPORT \& EXERCISE ISSN 1988-5202

(c) Faculty of Education. University of Alicante doi:10.14198/jhse.2019.14.Proc4.23 


\section{INTRODUCTION}

Aging is a physiological state of an organism that shows a great threat of dementia, fall, incontinence, weight loss, a sense of weakness and motor difficulties. In the last decades, attention to the elderly has been increased in various disciplines, as consequence of the demographic changing, given by the progressive aging of the population and by the growing number of elderly people in the industrialized countries (Sgrò et al., 2015). The elderly subjects are essentially represented in some categories: subjects with a low social and economic level, subjects whose physical activity undergoes a gradual reduction with age. Educational research has developed a renewed focus on older people. The multidisciplinary psychological, sociological proposals have underlined the multiplicity and variety of the evolutionary and existential dimensions of the elderly, so they require Pedagogy, and particularly, the Pedagogy of the course of life, to consider the experience of aging for its development. For these reasons there is the idea of promoting the resources of the elderly on the basis of lifelong learning: on one hand, there is a cultural change to overcome old stereotypes and undue social homologations; on the other hand, it is necessary to provide educational experiences to stimulate all the dimensions (such as cognitive, affective, relational, spiritual) of the whole aging person to realize wellbeing and social integration (Tiziana et al., 2017). The subjective and collective opportunities, available for the elderly in Europe, are increasing and diversifying at the present day, particularly, the segment of the population aged 65 to 74 is at the centre of the changes from an existential point of view as well as from a social point of view. (Boccacin, et al., 2014). Physical activity (PA) in old people has a fundamental importance in preventing diseases, maintaining independence and improving the quality of life. (Sun, et al., 2013). It is well known that regular physical activity of mild intensity leads to significant benefits in terms of health, while a sedentary lifestyle contributes, together with other risk factors, to the development of chronic degenerative diseases, particularly cardiovascular (Altavilla et al., 2018) metabolic and osteo-articular diseases (Vitulli, et al., 2012). The health of older people is the result of a complex interaction of causes, both in the present and in the heritage of the lifestyles of the past: economic, social, cultural, political, environmental, behavioural and biological factors contribute to favour or damage health(Gaetano, 2016). Even an insufficient supply of social care and of health services, it can have a more or less decisive impact on the onset and on the persistence of discomfort. In all phases of life, the state of health (psychic, physical and functional) positively or negatively affects the possibility of expression and construction of his own life, both individual, family and social level, at the material level (partner conditions) and at an immaterial level (participation in social life, relationships, subjective well-being, psychological wellbeing) (Sgrò et al., 2016; Sgrò et al., 2017). The 2015 ISTAT data show that $11.2 \%$ of the population perform regular physical activity. With reference to the elderly, the improvements, after nine months of motor activity with a frequency of 2 times a week for a period of $45-60$ minutes, that can be obtained and be classified as follows: development and conservation of the skeleton, maintenance and improvement of the joint function, preservation and improvement of postural muscle tone, improvement of the improvement of the cardiocirculatory function in the pulmonary function (Calcerano, et al., 2003). Those who practice regular physical activity (Raiola, 2017, 2013) lead a healthier lifestyle, eat better and try to avoid weight gain. In this regard, the Gerontology Society has proposed tests that allow the assessment of coordinative and conditional motor skills in the elderly from 65 to 75 to identify any deficiencies.

\section{METHODS}

\section{Participants and procedures}

$n=20$ ) participants: 10 women and 10 men they have been divided into two groups: 5 men and 5 women belonging to the experimental group, recruited in the gym and 5 men and 5 women belonging to the control group, who don't carry out physical activity. All training sessions were managed by accredited coaches who 
had more than five years coaching experience and specialized in Motor Sciences. The written informative consent was obtained from all the participants after a detailed explanation of the purpose of the study and of the description of the experimental methodology.

The first procedures of assessment were performed in the centre of the gymnasium in Catania, in September, while the second procedures of assessment were conducted in May in the same place and respecting the initial conditions. The training session was organized in a gym equipped with windows to change the air and a stereo system for the dissemination of music during the lesson, the subjects who trained in the morning from 10:00 to 11:00 for 9 months (three times a week / 60 minutes for each time).

The subjects were trained in the morning from 10:00 to 11:00. For the research, validated tests in geriatrics were used, in particular:

1. "Scale Tinetti Gait and Balance": The Tinetti scale is a simple test that measures the characteristics associated with falls: in this test the highest the score, and the best performance, the test can be administered in the gym or at home by health professionals. The version presented here is validated by the Geriatric Research Group Brescia (Rozzini, L et al., 2007; Bianchetti, A et al., 1990). The scale can be used to define the persons who must undergo special surveillance and rehabilitation programs and also to define the effectiveness or negative side effects of treatment and rehabilitation programs. "Tinetti Gait and Balance Scale" includes: Balance: which consists of 9 items with a score that can vary from 0 to a maximum of 16 . The subject must be evaluated while sitting on a chair without arms and is invited to perform the indicated manoeuvre. The quality of the response is evaluated, while the speed is not particularly relevant.

Walk: consists of 7 items with a score between 0 and 12. The subject is standing in front of the examiner, walking in the corridor or across the room, at the beginning with the usual step, then with a step a little faster. You can use the usual walking aids if necessary.

The evaluation of the performance is defined by measuring of the capacity of the subject according to the following scores:

$0=$ inability to execute what is the request.

$1=$ ability to execute it, but with adaptation.

2 = ability to perform it, without adaptation.

2. "Up \& Go Test" for the evaluation of general motility. It is measured in seconds, the time needed for the patient to get up from a chair (about $0.46 \mathrm{~m}$ in height), cover the distance of 3 meters, turn around a cone and sit down again. The subject wears comfortable shoes and can use his normal clothes. At the beginning of this test the back is in contact with the back. The subject should try to make the entire journey as quickly as possible without aid. The teacher's presence is important in case the subject loses balance. In fact, many seniors show difficulty with the coordination space - the time to get around the obstacle especially in the first tests. 11-20 seconds are normal for the elderly and disabled patients; times greater than 20 seconds indicates that the person needs the assistance and the further examination and intervention; score of 30 seconds or more suggests that the person may be prone to falls.

3. "30 seconds." Chair-Stand Test" for the indirect evaluation of the strength of the lower limbs. The subject is sitting on a chair of about $0.42 \mathrm{~m}$ (he is leaning against the wall) with his arms crossed over his chest. He 
is asked to stand up to the maximum extension of the knees and to sit on the chair continuously for 30 seconds, as fast as possible. The number of repetitions performed correctly is counted.

4. "30 seconds Arm Curl test" to evaluate the strength of the upper limbs (Jones, CJ et al., 1999). The test plans to flex the dominant arm towards itself the largest number of times using a handlebar to $2 \mathrm{~kg}$ for women and $3 \mathrm{~kg}$ for men. There is the risk of lack of autonomy both for women and for men when the handlebar is lifted for less than 11 times.

\section{Data analysis}

The statistical analysis has been performed using SPSS version 20.0 and were conducted at a significance level of $P<0.05$, except for the estimate effects. From the preliminary analysis of the data collected in the two evaluation sessions, the respect of the assumptions require for the univariate analysis has been studied. Not having been satisfied this assumption, to calculate is to identify the differences between the pre and post evaluations within the two groups the data were processed using nonparametric test for dependent samples. The latter has been calculated with the formula $r=Z / \sqrt{N}$ (Rosenthal, 1994) with the value of $Z$ obtained through the Wilcoxon. The size of the effects was interpreted according to the Cohen (1977): .00 -.20 insignificant; $.21-.49$ low; .50-.79, moderate; > 80 , large.

\section{RESULTS}

The data analyses were conducted separately for the control group and for the experimental group. No statistically significant differences were found in the pre and comparison of the control group except for the test 3 characterized by a worsening of the results. (tab.1). Muscle-strength loss has been shown to be greater for lower limbs in comparison to upper limbs (Landers, KA et al; 2001). The decrease in strength seems to be related to increasing age and muscle-mass loss, it is also likely to be a consequence of more physical inactivity. (Sayer, AA et al; 2008). This was confirmed by the IPAQ results, which showed that older (70-80 years) people were less physically active in all segments (work-related, transportation, housework/gardening, and leisure-time activity). The combination of muscle-strength loss, lower levels of PA, is a result of the aging process represents the potential risk for decreased mobility. (Visser, M et al; 2005).

Table 1. Descriptive indicators in variables of the tests $\mathrm{T} 1, \mathrm{~T} 2, \mathrm{~T} 3, \mathrm{~T} 4$

\begin{tabular}{|cccccc|}
\hline & \multicolumn{2}{c}{ Pre } & \multicolumn{2}{c}{ Post } & COMPARISON \\
\hline & $\mathrm{M}$ & $\mathrm{SD}$ & $\mathrm{M}$ & $\mathrm{SD}$ & $\mathrm{p}$ \\
\hline test 1 & 9,20 & 1,47 & 9,00 & 1,49 &, 31 \\
test 2 & 12,60 & 2,02 & 12,50 & 2,06 &, 046 \\
test 3 & 17,40 & 8,63 & 18,20 & 8,24 &, 038 \\
test 4 & 12,00 & 3,68 & 11,50 & 3,95 &, 059 \\
test 5 & 16,90 & 5,34 & 16,90 & 5,34 & 1,00 \\
\hline
\end{tabular}

Legend: A M - arithmetic mean, SD - standard deviation, $p$ - level of significant.

Conversely there were statistically significant differences between pre- and post- test evaluations of the experimental group, in which improvements were shown in all the test proposed with a predominantly large effect size. (tab.2). 
Table 2. Descriptive indicators in variables of the tests $\mathrm{T} 1, \mathrm{~T} 2, \mathrm{~T} 3, \mathrm{~T} 4$

\begin{tabular}{|ccccccc|}
\hline & \multicolumn{2}{c}{ Pre } & \multicolumn{2}{c}{ Post } & \multicolumn{2}{c|}{ COMPARISON } \\
\hline & $\mathrm{M}$ & $\mathrm{SD}$ & $\mathrm{M}$ & $\mathrm{SD}$ & $\mathrm{r}$ & $\mathrm{p}$ \\
\hline test 1 & 10,00 &, 94 & 11,90 &, 316 & 0.87 &, 00 \\
test 2 & 14,10 & 1,44 & 15,80 &, 422 & 0.75 &, 01 \\
test 3 & 12,60 & 3,23 & 10,20 & 3,01 & 0.92 &, 00 \\
test 4 & 14,20 & 1,87 & 16,90 & 1,66 & 0.81 &, 01 \\
test 5 & 17,20 & 2,04 & 20,40 & 2,21 & 0.92 &, 00 \\
\hline
\end{tabular}

Legend: $A M$ - arithmetic mean, $S D$ - standard deviation, $r=$ 'effect size (.00 -.20 insignificant; .21-.49 low; .50-.79, moderate; $>.80$, large), , $p-$ level of significant.

\section{DISCUSSION}

The results of the research showed that there are differences between the pre and post training of the experimental group that participated in the research. This confirms that the research aim have been achieved and that physical activity improves the quality of life of older people; a very important factor as the aging of the population is one of the most important social phenomena of the coming decades. Therefore, the need arises to guarantee the elderly the opportunity to live their years in the best possible way or without unfavourable health conditions. To achieve these goals, it is necessary to promote the physical activity of health workers, involving different sectors and working in collaboration with various professional figures of physical activity. The results of this research are in agreement with the results obtained so far from previous studies (Bicego et al., 2009; Brown et al., 2004, Vuillemin et al., 2005). Previous studies have shown that different types of PAs such as treadmill (Oh-Park, et al., 2011), stretching exercises (Cristopoliski, et al., 2009) and Pilates (Newell, et al., 2012) are able to provide beneficial effects on gait, albeit with variable values of improvement. Furthermore, our results seem to suggest that only when the muscles of the lower limbs are sufficiently stimulated by training can a positive effect be found on gait patterns. This is somewhat consistent with the results (Persch et al., 2009) who reported improvements in gait kinematics after a 12week strength training program and associated these changes with greater strength, particularly in muscle groups around the knee. It is certainly very important to pay attention to the details that can influence the test results (Miketa, et al., 2017).

\section{CONCLUSION}

The study carried out shows some aspects related to the application of a program of physical activity in the elderly. In the first place, the general purpose was reached, as it was possible to involve older persons of both sexes in a motor activity program, through the collaboration of colleagues of physical sciences who actively collaborated in the identification of subjects and in the evaluation phase, thus bringing concrete and important support to the initiative. Our initial hypotheses were confirmed, in fact we found differences even if not statistically significant among the groups that participated in the study. Naturally the results obtained refer to a very low sample of the population and it would be more correct to extend the research to a greater number to verify the reliability of the tests proposed. but at present, we can state that the results obtained, show how the development of an appropriate training protocol can bring benefits in the daily life activities of older people. so physical activity must be undertaken as a global stimulus for the whole body and adapted to the cultural, creative, and emotional possibilities of the subject. Exercise is useful for increasing gait speed, improving balance and improving performance in the frailest adults (Chou et al., 2012). This information can be useful in planning specific PA protocols to the needs of each individual, also in consideration of the desired 
goal and health / training conditions. Physical activity in the elderly of human beings always favours a global activation of the organism, positively influencing the aging process and strengthening adaptation. So we need a greater awareness that leads to an authentic re-foundation of our country's way of thinking.

\section{REFERENCES}

Aihie-Sayer A, Dennison EM, Syddall HE, Jameson K, Martin HJ, Cooper C. The developmental origins of sarcopenia: using peripheral quantitative computed tomography to assess muscle size in older people. J Gerontol A Biol Sci Med Sci. 2008;63(8):835-840. https://doi.org/10.1093/gerona/63.8.835

Altavilla, G., D'Elia, F., Raiola, G. (2018) A brief review of the effects of physical activity in subjects with cardiovascular disease: An interpretative key Sport Mont, 16 (3), pp. 103-106.

Bianchetti, A., Rozzini, R., Carabellese, C., Zanetti, O., \& Trabucchi, M. (1990). Nutritional intake, socioeconomic conditions, and health status in a large elderly population. Journal of the American Geriatrics Society, 38(5), 521-526. https://doi.org/10.1111/.1532-5415.1990.tb02401.x

Bicego, D., Brown, K., Ruddick, M., Storey, D., Wong, C., \& Harris, S. R. (2009). Effects of exercise on quality of life in women living with breast cancer: a systematic review. The breast journal, 15(1), 4551. https://doi.org/10.1111/j.1524-4741.2008.00670.x

Brown, D. W., Brown, D. R., Heath, G. W., Balluz, L., Giles, W. H., Ford, E. S., et al. (2004). Associations between physical activity dose and health-related quality of life. Medicine and Science in Sports and Exercise, 36(5), 890- 896. https://doi.org/10.1249/01.mss.0000126778.77049.76

Casolo, F., Calcerano, L. (2007). Educazione motoria e sportiva (II ed.), Mondadori: La Scuola, 33- 35.

Ćešković, T., \& Kuzmanić, B. (2014, January). Assessment of balance in elderly people. In Scientific Conference" FIS Communications 2014". At Niš, Serbia.

Chou, C. H., Hwang, C. L., \& Wu, Y. T. (2012). Effect of exercise on physical function, daily living activities, and quality of life in the frail older adults: a meta-analysis. Archives of physical medicine and rehabilitation, 93(2), 237-244. https://doi.org/10.1016/i.apmr.2011.08.042

Cohen, J. (1988). Statistical power analysis for the behavioral sciences. Elsevier, New York, 2nd.

Gaetano, A. (2016) Relationship between physical inactivity and effects on individual health status, Journal of Physical Education and Sport, 16, pp. 1069-1074.

Gastaldi, A., \& Contarello, A. (2006). Una questione di età: rappresentazioni sociali dell'invecchiamento in giovani e anziani. Ricerche di Psicologia, 4 (1), 65 - 66.

Jones, C. J., Rikli, R. E., \& Beam, W. C. (1999). A 30-s chair-stand test as a measure of lower body strength in community-residing older adults. Research quarterly for exercise and sport, 70(2), 113119. https://doi.org/10.1080/02701367.1999.10608028

Landers KA, Hunter GR, Wetzstein CJ, Bamman MM, Weinsier RL. (2001) The interrelationship among muscle mass strength and the ability to perform physical tasks of daily living in younger and older women. J Gerontol A Biol Sci Med Sci. 56(10), B443-B448. [PubMed]. https://doi.org/10.1093/gerona/56.10.b443

Marsili, M., \& Sorvillo, M. P. (2002). Previsioni della popolazione residente per sesso, età e regione dal 1.1. 2001 al 1.1. 2051. Istituto Nazionale di Statistica, ISTAT, Roma.

Macchi, C. \& Molino Lova, R. (2007). Attività Motoria Fitness e Salute nell'adulto e nell'anziano (II ed.). Nicodemus Maggiulli Publisher, Florence, 373-379.

Miketa, T., Ivančić, N., \& Kuzmanić, B. (2017). Relationship of breathing exercises with improvement of postural stability in healthy adults. Acta Kinesiologica, 11(2), 59-62.

Newell, D., Shead, V., \& Sloane, L. (2012). Changes in gait and balance parameters in elderly subjects attending an 8-week supervised Pilates programme. Journal of bodywork and movement therapies, 16(4), 549-554. https://doi.org/10.1016/j.jbmt.2012.02.002 
Pichierri, M., \& Guido, G. (2017). Anziani e social media: opportunità o minaccia?. Salute e società, 3, 10. https://doi.org/10.3280/ses2017-003012

Podsiadlo, D., \& Richardson, S. (1991). The timed "Up \& Go": a test of basic functional mobility for frail elderly persons. Journal of the American geriatrics Society, 39(2), 142-148. https://doi.org/10.1111/i.1532-5415.1991.tb01616.x

Persch, L. N., Ugrinowitsch, C., Pereira, G., \& Rodacki, A. L. (2009). Strength training improves fallrelated gait kinematics in the elderly: a randomized controlled trial. Clinical Biomechanics, 24(10), 819-825. https://doi.org/10.1016/i.clinbiomech.2009.07.012

Raiola, G. (2017) Motor learning and teaching method, Journal of Physical Education and Sport, 17, art. no. 236, pp. 2239-2243.

Raiola, G. (2013) Body knowledge and motor skills, Knowledge Cultures, 1 (6), pp. 64-72.

Rosenthal, R. (1994). Parametric measures of effect size. The handbook of research synthesis, 621, 231-244.

Rozzini, L., Costardi, D., Chilovi, B. V., Franzoni, S., Trabucchi, M., \& Padovani, A. (2007). Efficacy of cognitive rehabilitation in patients with mild cognitive impairment treated with cholinesterase inhibitors. International Journal of Geriatric Psychiatry: A journal of the psychiatry of late life and allied sciences, 22(4), 356-360. https://doi.org/10.1002/gps.1681

Rodacki, A. L., Souza, R. M., Ugrinowitsch, C., Cristopoliski, F., \& Fowler, N. E. (2009). Transient effects of stretching exercises on gait parameters of elderly women. Manual therapy, 14(2), 167-172. https://doi.org/10.1016/..math.2008.01.006

Sgrò, F., Licari, D., Coppola, R., \& Lipoma, M. (2015). Assessment of balance abilities in elderly people by means of a clinical test and a low-cost force plate. Kinesiology: International journal of fundamental and applied kinesiology, 47(1), 33-43.

Sgrò, F., Quinto, A., Pignato, S., \& Lipoma, M. (2016). Comparison of product and process oriented model accuracy for assessing countermovement vertical jump motor proficiency in pre-adolescents. Journal of Physical Education and Sport, 16(3), 921-927.

Sgrò, F., Quinto, A., Messana, L., Pignato, S., \& Lipoma, M. (2017). Assessment of gross motor developmental level in Italian primary school children. Journal of Physical Education and Sport, 17(3), 1954-1959.

Tàmmaro, A. E., Cesana, L., \& Decio, G. (2008). Attività fisica, salute e cognitività nell'anziano. Ricerche di Psicologia, 1(1-2), 75.

Tinetti, M. E. (1986). Performance-oriented assessment of mobility problems in elderly patients. Journal of the American Geriatrics Society, 34(2), 119-126. https://doi.org/10.1111/j.1532$\underline{5415.1986 . t b 05480 . x}$

Tiziana, D., Antonetta, M., Gaetano, A. (2017) Health and physical activity [Zdravlje i tjelesna aktivnost]Sport Science, 10 (1), pp. 100-105.

Verghese, J., Holtzer, R., Oh-Park, M., Derby, C. A., Lipton, R. B., \& Wang, C. (2011). Inflammatory markers and gait speed decline in older adults. Journals of Gerontology Series A: Biomedical Sciences and Medical Sciences, 66(10), 1083-1089. https://doi.org/10.1093/gerona/glr099

Visser M, Goodpaster BH, Kritchevsky SB, et al. Muscle mass muscle strength and muscle fat infiltration as predictors of incident mobility limitations in well-functioning older persons. J Gerontol A Biol Sci Med Sci. 2005;60(3):324-333. https://doi.org/10.1093/gerona/60.3.324

Vitulli, P., Femminella, G. D., Ciccarelli, A. L., Rengo, F., Lombardi, A., Cellurale, M., Rengo, F. (2012). Attività fisica e invecchiamento. G. Geront, 60, 172-181.

Vuillemin, A., Boini, S., Bertrais, S., Tessier, S., Oppert, J.- M., Hercberg, S., et al. (2005). Leisure time physical activity and health-related quality of life. Preventive Medicine, 41(2), 562-569. https://doi.org/10.1016/j.ypmed.2005.01.006 


\section{(c) $(i) \Theta \Theta$}

This work is licensed under a Attribution-NonCommercial-NoDerivatives 4.0 International (CC BY-NC-ND 4.0). 